\title{
Alterations in the conjunctival bacterial flora following a single dose of azithromycin in a trachoma endemic area
}

Kenneth C Chern, S K Shrestha, Vicky Cevallos, H L Dhami, Pawan Tiwari, Laurie Chern, John P Whitcher, Thomas M Lietman

\begin{abstract}
Backgroundlaims-The World Health Organisation has recommended repeated mass treatment of children in trachoma endemic areas with oral azithromycin. While chlamydia, the causative agent of trachoma, remains universally sensitive to azithromycin, there is concern that large scale programmes may alter the bacterial flora and induce resistance in streptococcal species. In this study the effect of a single dose of azithromcyin on the prevalence, species distribution, and resistance of conjunctival bacterial flora was determined.
\end{abstract}

Methods-Baseline and 14 day follow up bacterial cultures were taken from the conjunctivae of 121 children who reside in a trachoma endemic area of Nepal. 91 children were treated with azithromycin at baseline and 31 children received deferred treatment at the $\mathbf{1 4}$ day follow up. Results-Although the prevalence of bacterial pathogens decreased significantly with azithromycin treatment, a significant change in the distribution of specific bacterial pathogens could not be demonstrated. Streptococcal resistance to azithromycin was found significantly more frequently after treatment. No change in the prevalence, distribution, or resistance pattern was found in the untreated control group.

Conclusion-Repeated mass treatment of trachoma endemic areas with oral azithromycin will have an effect on bacterial flora. However, further work needs to be done to determine if this will have any clinical relevance.

(Br f Ophthalmol 1999;83:1332-1335)

Lumbini Rana-Ambika Eye Hospital, Bhairahawa, Nepal

S K Shrestha

H L Dhami

P Tiwari

St Margaret's Hospital, Pittsburgh, PA, USA

L Chern

Correspondence to:

Thomas Lietman, MD

Francis I Proctor

Foundation, University of California, San Francisco, 95

Kirkham Street, Box 0944

San Francisco, CA

94143-0944, USA

Accepted for publication 20 July 1999 antibacterial activity against many bacterial species including pathogenic bacteria that are routinely found in the conjunctiva. While Chlamydia has remained sensitive to macrolides and azalides including azithromycin, other bacteria may develop resistance, and there is some concern that large scale trachoma programmes may interfere with bacterial flora. ${ }^{3}$ In this study, we investigated the effect of azithromycin on the bacterial flora of children in a trachoma endemic area. In particular, we addressed the following questions. Does a single dose of azithromycin decrease the prevalence of conjunctival bacteria? Does a single dose of azithromycin alter the distribution of bacterial species found in the conjunctival flora? Does a single dose of azithromycin result in resistant Streptococcus pneumoniae or Haemophilus influenzae in the conjunctiva?

Trachoma is the second leading cause of blindness worldwide and is the leading cause of infectious blindness. ${ }^{4}$ Recurrent infection of children with $C$ trachomatis can lead to a cascade of conjunctival scarring, trichiasis, corneal ulceration, and ultimately blindness. Trachoma is endemic in poor, arid areas of Africa and Asia. The WHO has declared Nepal a high priority country for trachoma control. ${ }^{1}$ The Lumbini zone in mid-western Nepal is an area known from previous studies to have endemic trachoma. ${ }^{56}$

Azithromycin is an azalide antibiotic with a broad spectrum of activity against Gram positive and Gram negative bacteria. It has unique pharmacokinetic properties including a long half life, and concentration of the antibiotic in local tissue reservoirs. ${ }^{7}$ After oral dosing, tissue specimens from the conjunctiva have concentrations of azithromycin over 100 times the level in the serum. ${ }^{8}$ Azithromycin is also more highly concentrated in inflamed tissues such as the sinuses, ${ }^{9}$ which may be due, in part, to the preferential accumulation of azithromycin by polymorphonuclear leucocytes and other phagocytic cells. ${ }^{10}$ Moreover, the minimum inhibitory concentration of azithromycin in conjunctival tissue remains above that necessary to inhibit $C$ trachomatis after 6 days. ${ }^{11} \mathrm{By}$ the eighth day following oral dosing, the serum drug level is undetectable while the tissue levels remained above the MIC90 for $S$ pneumoniae and $H$ influenzae. ${ }^{12}$

Azithromycin has in vitro activity against many bacterial species commonly found on the conjunctiva, including $S$ pneumoniae, H influenzae, Staphylococcus species, $S$ pyogenes, and $C$ trachomatis. ${ }^{7}$ The low incidence of side effects and the high compliance with the dosing 
schedule make azithromycin an ideal drug for public health purposes. A single oral dose of azithromycin has been found to be as effective against active trachoma as $4-6$ weeks of topical tetracycline ointment. ${ }^{213}$

\section{Materials and methods \\ STUDY POPULATION}

A village and a nearby elementary school in the Lumbini zone of mid-western Nepal were chosen by recommendation of the local healthcare officials. Informed consent was obtained for the children through consultation with guardians and the village chief. Baseline and 14 day follow up evaluations were performed on children aged 1-10 years at each location. On the baseline visit, trained examiners screened children for evidence of early trachoma by grading the right upper conjunctiva according to the simplified WHO grading scale for trachoma. ${ }^{14}$ Children were considered clinically active if their right conjunctivae were scored as follicular trachoma (TF) or intense trachoma (TI). The right upper conjunctivae of all children were swabbed for bacterial cultures on blood agar plates. Ninety one children were randomised to receive oral azithromycin (Zithromax paediatric suspension, Pfizer International) at a dose of $20 \mathrm{mg} / \mathrm{kg}$ (the treated group) at baseline, and 31 children were randomised to receive the same dose of azithromycin deferred until the 14 day follow up visit (the control group). Randomisation (by choosing marked cards from a shuffled deck) was performed after the examination and culture. There was no use of antibiotic ointment or any new introduction of lid hygiene between baseline and the 14 day follow up.

Table 1 Characteristics of treated and control groups

\begin{tabular}{llll}
\hline & Treated & Control & Total \\
\hline Children examined at baseline visit & 91 & 31 & 122 \\
Children examined at 14 day visit & 84 & 25 & 109 \\
Mean age at baseline visit & 7.1 & 7.0 & 7.1 \\
Active trachoma at baseline visit & $35(39 \%)$ & $11(35 \%)$ & $46(38 \%)$ \\
\hline
\end{tabular}

*Active trachoma is defined as follicular trachoma (TF) or intense trachoma (TI) on clinical examination according to the WHO simplified trachoma grading scale. ${ }^{13}$

Table 2 Prevalence of pathogenic bacteria ${ }^{\star}$

\begin{tabular}{lllll}
\hline \multicolumn{3}{c}{ Baseline visit } & \multicolumn{3}{c}{14 day visit } \\
\hline Treated & $36 / 91$ & $(40 \%)$ & $13 / 84$ & $(15 \%)$ \\
Control & $5 / 31$ & $(16 \%)$ & $4 / 25$ & $(16 \%)$ \\
Total & $41 / 122$ & $(34 \%)$ & $17 / 109$ & $(16 \%)$ \\
\hline
\end{tabular}

^Pathogenic bacteria are defined, for the purposes of this study, as Staphylococcus aureus, Streptococcus pneumoniae, Haemophilus influenzae, Moraxella $\mathrm{sp}$, Neisseria $\mathrm{sp}$, and other Gram negative rods such as Escherichia coli.

Table 3 Bacterial species isolated

\begin{tabular}{|c|c|c|c|c|c|c|}
\hline & \multicolumn{3}{|c|}{ Baseline visit (122 children examined) } & \multicolumn{3}{|c|}{14 day visit (109 children examined) } \\
\hline & Treated & Control & Total & Treated & Control & Total \\
\hline S pneumoniae & 25 & 2 & 27 & 7 & 3 & 10 \\
\hline Haemophilus & 8 & 1 & 9 & 5 & 1 & 6 \\
\hline Moraxella & 4 & 2 & 6 & 0 & 0 & 0 \\
\hline Neisseria & 2 & 0 & 2 & 0 & 0 & 0 \\
\hline E coli & 0 & 0 & 0 & 1 & 0 & 1 \\
\hline Total & 39 & 5 & 44 & 13 & 4 & 17 \\
\hline
\end{tabular}

The conjunctivae of some children had growth of more than one bacterial species.
LABORATORY ANALYSIS

The bacterial cultures were incubated and processed using standard microbiological techniques at the microbiology laboratory at the Lumbini-Rana Eye Hospital in Bhairahawa, Nepal. A dot of Staphylococcus epidermidis was placed on each plate to allow for growth of Haemophilus species. ${ }^{15}$ Pathogenic bacteria (defined for the purposes of this study as Staphylococcus aureus, Streptococcus pneumoniae, Haemophilus influenzae, Moraxella sp, Neisseria sp, and other Gram negative rods such as Escherichia coli) were isolated for identification and further testing. Antibiotic sensitivity for azithromycin and penicillin $G$ was performed using the E-Test (AB Biodisk, Sweden) on all isolates of $S$ pneumoniae and $H$ influenzae.

Fourteen days after the initial visit, we returned to the school and village and recultured the conjunctivae of the same children who were previously examined. Children randomised to deferred azithromycin treatment were treated at this time.

\section{DATA ANALYSIS}

The $\chi^{2}$ statistic was used to compare the baseline and follow up prevalence of bacteria, distribution of bacteria, and prevalence of $S$ pneumoniae resistance. When the expected number in a cell was five or fewer, a Fisher's exact test was used.

\section{Results}

PATIENT POPULATION

On the first visit, we examined 54 children at the school and 68 children at the village. The children ranged in age from 6-10 years of age in the school and 1-10 years of age in the village, with an overall average age of 7.1 years. Ninety one $(75 \%)$ of the 122 children were randomised to receive azithromycin treatment at the first visit. One hundred and nine (89\%) of the 122 children examined on the first visit were located and recultured on the 14 day visit (Table 1).

\section{TRACHOMA GRADING}

In total, $46(38 \%)$ of 122 of children were graded as clinically active; $11(20 \%)$ of 54 from the school were active, and $35(51 \%)$ of 68 children in the village. The proportion of active cases in the treated ( 35 of 91 , or $38 \%$ ) and in the control group (22 of 31 , or $37 \%$ ) were not significantly different ( $p>0.90, \chi^{2}$ test).

\section{CULTURE RESULTS}

Pathogenic bacteria were recovered from the conjunctivae of $36(40 \%)$ of 91 children in the treated group on the initial visit (Table 2). At the 14 day follow up, 13 (18\%) of the 84 children had pathogenic bacteria isolated from their conjunctiva. There was a statistically significant decline in the number of pathogenic bacteria from before to after azithromycin treatment $\left(p<0.01, \chi^{2}\right.$ test). In the control group, the percentage of children with pathogenic bacteria remained unchanged in the 2 week period.

The distribution of bacterial isolates in the school and village is summarised in Table 3. In 
Table 4 Children with recovery of bacteria on initial and subsequent cultures

\begin{tabular}{|c|c|c|c|c|c|}
\hline \multirow[b]{2}{*}{ Baseline culture } & \multicolumn{2}{|l|}{ Sensitivity to: } & \multirow[b]{2}{*}{14 day culture } & \multicolumn{2}{|l|}{ Sensitivity to: } \\
\hline & Azithromycin & Penicillin & & Azithromycin & Penicillin \\
\hline Haemophilus sp & Sensitive & Not tested & Haemophilus sp & Sensitive & Not tested \\
\hline$S$ pneumoniae & Sensitive & Sensitive & $S$ pneumoniae & Sensitive & Sensitive \\
\hline S pneumoniae & Sensitive & Sensitive & $S$ pneumoniae & Sensitive & Sensitive \\
\hline S pneumoniae & Sensitive & Sensitive & $S$ pneumoniae & Sensitive & Sensitive \\
\hline S pneumoniae & Sensitive & Sensitive & S pneumoniae & Sensitive & Sensitive \\
\hline S pneumoniae & Sensitive & Sensitive & S pneumoniae & Resistant & Sensitive \\
\hline$S$ pneumoniae & Sensitive & Sensitive & S pneumoniae & Resistant & Sensitive \\
\hline
\end{tabular}

the treated group, $S$ pneumoniae was isolated from 25 children; Haemophilus species in eight children, Moraxella species in four children, and Neisseria species in two children. At the 14 day visit, $S$ pneumoniae was again the most frequent isolate occurring in seven patients; Haemophilus species in five patients; and $E$ coli in one patient. The difference in the distribution of organisms at baseline and at 14 days did not reach statistical significance $\left(p=0.10, \chi^{2}\right.$ test $)$. Also there was no significant difference in the distribution of bacterial species in the control group over the 14 day period.

\section{SENSITIVITIES}

Antibiotic sensitivities to azithromycin and penicillin $\mathrm{G}$ were performed on all isolates of $S$ pneumoniae using the E-test (AB Biodisk, Sweden). The Haemophilus species were tested for azithromycin sensitivity. Of the $27 \mathrm{~S}$ pneumoniae isolates tested from the first visit (from both the treated and the control groups), all were sensitive to both azithromycin and penicillin G. Likewise, all nine Haemophilus isolates were sensitive to azithromycin. From the 14 day collection in the control group, all three isolates of $S$ pneumoniae remained sensitive to the antibiotics. However, in the treated group, three of seven isolates of $S$ pneumoniae were resistant to azithromycin. There was no change in sensitivity in these isolates to penicillin G. The five Haemophilus isolates found on the day 14 visit were sensitive to azithromycin.

PAIRED CULTURE RESULTS

Seven children had growth of the same bacterial species on the initial and subsequent culture (Table 4). Six of the seven children grew $S$ pneumoniae on both cultures and one child grew Haemophilus species. Whereas all initial $S$ pneumoniae isolates were sensitive to azithromycin by E-test, two isolates from the second visit were resistant to this antibiotic. All isolates were sensitive to penicillin $G$. The Haemophilus species was sensitive to azithromycin both before and after treatment.

\section{Discussion}

In the patients treated with azithromycin, we found a statistically significant decrease in the recovery of pathogenic bacteria from the conjunctiva, from $39 \%$ to $14 \%$. Patients in the untreated control group had no change in the number of positive cultures over this 14 day time period. The systemic administration of azithromycin has also been shown to decrease bacterial flora elsewhere in the body. Adegbola et $a l^{16}$ reported a decrease of $S$ pneumoniae recoverable from the oropharynx from $73 \%$ to $24 \%$ in children after azithromycin treatment. Leach et $a l^{17}$ also found a statistically significant decrease in oropharyngeal $S$ pneumoniae from $68 \%$ at baseline to $29 \% 2$ weeks after azithromycin dosing. At 2 and 6 months after treatment, however, the rates of positive bacterial cultures had returned to baseline. The lower rate of $S$ pneumoniae found in our study may be because of the culture source (conjunctiva rather than oropharynx), the study population (Leach et al sampled only children with active trachoma, who may tend to be at an age or in a socioeconomic group more likely to harbour pneumococcus as well), or the study location and season.

The most frequent pathogenic bacteria isolated in this study were $S$ pneumoniae and $H$ influenzae, and Moraxella. The prevalence of all three pathogens was reduced after azithromycin treatment. The distribution of bacterial species was not statistically different at baseline and 14 days after treatment. Previous studies of trachomatous eyes also had found $S$ pneumoniae and $H$ influenzae to be the most common non-chlamydial ocular pathogen. ${ }^{18}$ Adegbola et $a l^{16}$ found that $S$ pneumoniae was less prevalent after azithromycin treatment, but that $H$ influenzae was not significantly affected. In many countries, seasonal variation plays an important role in both the number and distribution of bacteria, as suggested by Peterson and Treadway. ${ }^{19}$ It should be noted that the effect of azithromycin on the flora may also change during the course of the year, so care should be taken when generalising these results. Seasonal variation between the baseline and the follow up collections was relatively short (14 days) and was not thought to be a factor in the change in flora. In fact, there was no significant change in the frequency of bacterial infection in the control group, although the power to detect such a change was dependent on the relatively small size of this group.

On the 14 day follow up visit, we found that three of seven isolates of $S$ pneumoniae from the treated group were resistant to azithromycin in vitro. Previously, two of these patients had on their initial cultures growth of $S$ pneumoniae which was sensitive to azithromycin. The third patient initially had an $\alpha$ haemolytic colony which could not be isolated from the background contamination, and in retrospect, may also have been $S$ pneumoniae. No resistant isolates were present in the control group. Moreover, there was no change in the sensitivity of 
the $S$ pneumoniae isolates to an unrelated class of antibiotics, penicillin G. Leach et $a l^{17}$ also found an increase in azithromycin resistant $S$ pneumoniae from $1.3 \%$ to $15.8 \%$ following a single dose of azithromycin. In a large Finnish study, ${ }^{20}$ higher rates of macrolide resistant $S$ pyogenes occurred in parallel with the increased prescription of macrolide and azalide antibiotics at a national level. As the use of macrolides and azilides was reduced resistance decreased.

For several reasons, it is not known whether or not the acquired resistance found in this study is clinically relevant. Most colonising bacteria (resistant or sensitive) will not go on to cause a productive infection. Productive infections are often not treated with macrolide or azalide antibiotics, but instead with antibiotics to which $S$ pneumoniae will presumably remain sensitive. Resistance found in vitro often does not correlate with clinical resistance. Resistant organisms tend to be less fit than sensitive organisms, presumably because they have had to make a compromise to become resistant, thus they may be less likely to cause infection and less likely to spread to other individuals ${ }^{21}$; it is not known how often resistant bacteria colonising either the conjunctiva or the oropharynx will produce infections in others (that is, cause initial resistance). The resistance may be transient, and it may be reversible when treatment programmes conclude, as suggested in Australian and Finnish studies. ${ }^{17} 20$

Mass treatment with azithromycin is recommended by the WHO for the treatment of endemic trachoma, and treatment programmes are already under way in some countries. We expect the use of any systemic antibiotic (including azithromycin) to have an effect on bacterial flora, both decreasing bacterial flora and increasing drug resistance in bacteria such as $S$ pneumoniae. There is a large global programme already in place that monitors the development of resistance to azithromycin. ${ }^{1}$ It will be particularly important to monitor the prevalence of resistance in areas receiving repeated mass administrations of azithromycin, in actual infections (not just in colonisation), and in both individuals who have received azithromycin (acquired resistance) and those who have not (initial resistance).
We thank Suzanne Gilbert, PhD, R P Pokhrel, MD, Charles Leiter, Morton Leiter, and Susan Osaki, MPH for their considerable assistance with this project. We are grateful to the National Institute of Allergy and Infectious Diseases (Grant K08 AI 01441), Pfizer International Inc, and the Edna McConnell Clark Foundation for their generous support.

1 World Health Organisation. Report of the first meeting of the WHO alliance for the global elimination of trachoma. Geneva: WHO, 1997.

2 Bailey RL, Arullendran P, Whittle HC, et al. Randomised controlled trial of single-dose azithromycin in treatment of trachoma. Lancet 1993;342:453-6.

3 De Sole G. Elimination of trachoma: follow up (letter). $\mathrm{Br} \mathcal{F}$ Ophthalmol 1998;82:590.

4 Thylefors B. A global initiative for the elimination of avoidable blindness. Am f Ophthalmol 1998;125:90-3.

5 Brilliant G. The epidemiology of blindness in Nepal: report of the 1981 Nepal blindness survey. Chelsea, MI: Seva Fondationa, 1988.

6 Lietman T, Dhital S, Dena D. Conjunctival impression cytology for vitamin A deficiency in the presence of infectious trachoma. Brf Ophthalmol 1998;82:1139-42.

7 Lode H, Borner K, Koeppe P, et al. Azithromycin - review of key chemical, pharmacokinetic and microbiological features. F Antimicrob Chemother 1996;37(Suppl C) 1 .

8 Tabbara K, al-Kharashi S, al-Mansouri S, et al. Ocular levels of azithromycin. Arch Ophthalmol 1998;116:1625-8.

9 Karma P, Pukander J, Penttilä M. Azithromycin concentrations in sinus fluid and mucosa after oral administration Eur f Clin Microbiol Infect Dis 1991;10:856-9.

10 Wildfeuer A, Laufen H, Zimmermann T. Distribution of orally administered azithromycin in various blood compartments. Int f Clin Pharmacol Ther 1994;32:356-60.

11 Karcioglu ZA, El-Yazigi A, Jabak MH, et al. Pharmacokinetics of azithromycin in trachoma patients: serum and tear levels. Ophthalmology 1998;105:658-61.

12 Vaudaux BP, Cherpillod J, Dayer P. Concentrations of azithromycin in tonsilar and/or adenoid tissue from paediatric patients. F Antimicrob Chemother 1996;37(Suppl C):45-51.

13 Tabbara KF, Abu-el-Asrar A, al-Omar O, et al. Single-dose azithromycin in the treatment of trachoma. A randomized, azithromycin in the treatment of trachoma. A ran

14 Thylefors B, Dawson CR, Jones BR, et al. A simple system for the assessment of trachoma and its complications. Bull World Health Organ 1987;65:477-83.

15 Smolin G, Tabbara K, Whitcher J. Infectious diseases of the eye. Baltimore: Williams and Wilkins, 1984.

16 Adegbola RA, Mulholland EK, Bailey R, et al. Effect of azithromycin on pharyngeal microflora (letter). Pediatr Infect Dis F 1995; 14:335-7.

17 Leach AJ, Shelby-James TM, Mayo M, et al. A prospective study of the impact of community-based azithromycin treatment of trachoma on carriage and resistance of Streptococcus pneumoniae. Clin Infect Dis 1997;24:356-62.

18 Whitcher JP, Dawson CR, Messadi M, et al. Severe endemic trachoma in Tunisia: changes in ocular bacterial pathogens in children treated by the intermittent antibiotic regimen. Revue Internationale du Trachome (International Review of Trachoma) 1974;51:49-58.

19 Peterson J, Treadway G. Impact of community-based azithromycin treatment of trachoma on carriage and resistance of Streptococcus pneumoniae (letter). Clin Infect Dis 1998;26:248-9.

20 Seppälä H, Klaukka T, Vuopio-Varkila J, et al. The effect of changes in the consumption of macrolide antibiotics on erythromycin resistance in group A streptococci in Finland. Finnish Study Group for Antimicrobial. $N$ Engl $\mathcal{F}$ Med 1997;337:441-6.

21 Stewart FM, Antia R, Levin BR, et al. The population genetics of antibiotic resistance. II: Analytic theory for sustained populations of bacteria in a community of hosts. Theor Popul Biol 1998;53:152-65. 